\title{
Propensity matched analysis of short term oncological and perioperative outcomes following robotic and thoracolaparoscopic esophagectomy for carcinoma esophagus- the first Indian experience
}

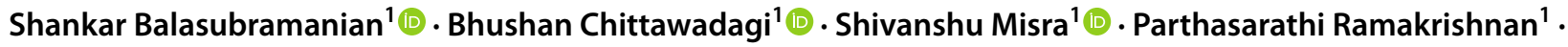 \\ Palanivelu Chinnusamy ${ }^{1}$
}

Received: 23 November 2020 / Accepted: 3 February 2021 / Published online: 20 February 2021

(c) The Author(s), under exclusive licence to Springer-Verlag London Ltd. part of Springer Nature 2021

\begin{abstract}
Thoracolaparoscopic esophagectomy (TLE) for carcinoma esophagus has better short-term outcomes compared to open esophagectomy. The precise role of robot-assisted laparoscopic esophagectomy (RALE) is still evolving. Single center retrospective analysis of TLE and RALE performed for carcinoma esophagus between January 2015 and September 2018. Propensity score matching was done between the groups for age, gender, BMI, ASA grade, tumor location, neoadjuvant therapy, the extent of surgical resection (Ivor Lewis or McKeown's), histopathological type (squamous cell carcinoma or adenocarcinoma), clinical $\mathrm{T}$ and $\mathrm{N}$ stages. The primary outcome parameter was lymph node yield. Secondary outcome parameters were resection margin status, duration of surgery, blood loss, conversion to open procedure, length of hospital stay, length of ICU stay, complications, 90-day mortality and cost. There were 90 patients in TLE and 25 patients in RALE group. After propensity matching, there were 22 patients in each group. The lymph node yield was similar in both the groups $(23.95 \pm 8.23$ vs $22.73 \pm 11.63 ; p=0.688)$. There were no conversions or positive resection margins in either group. RALE was associated with longer operating duration $(513.18 \pm 91.23 \mathrm{~min}$ vs $444.77 \pm 64.91 \mathrm{~min} ; p=0.006)$ and higher cost $(\$ 5271.75 \pm 456.46$ vs $\$ 4243.01 \pm 474.64 ; p<0.001)$ than TLE. Both were comparable in terms of blood loss $(138.86 \pm 31.20 \mathrm{ml}$ vs $133.18 \pm 34.80 \mathrm{ml} ; p=0.572)$, Clavien-Dindo grade IIIa and above complications (13.64\% vs $9.09 \%$; $p=0.634)$, hospital stay $(12.18 \pm 6.35$ days vs $12.73 \pm 7.83$ days; $p=0.801)$, ICU stay $(4.91 \pm 5.22$ days vs $4.77 \pm 4.81$ days; $p=0.929$ ) and mortality ( 0 vs $4.55 \% ; p=0.235$ ). RALE is comparable to TLE in terms of short-term oncological and perioperative outcomes except for longer operating duration when performed for carcinoma esophagus. RALE is costlier than TLE.
\end{abstract}

Keywords Carcinoma $\cdot$ Esophagus $\cdot$ Esophagectomy $\cdot$ Laparoscopy $\cdot$ Thoracoscopy $\cdot$ Robot

\section{Introduction}

Carcinoma esophagus is the fourth leading cause of cancerrelated deaths in India [1]. Patients with carcinoma esophagus are often nutritionally debilitated, and conventional open methods of esophagectomy with its attendant high morbidity make the management of this complex group of patients challenging [2]. The management of carcinoma esophagus has evolved considerably over the past two decades, with

Shankar Balasubramanian

shanxar@gmail.com

1 Department of Surgical Gastroenterology, GEM Hospital and Research Center, Coimbatore, India minimally invasive surgery playing an increasing role in treating this condition.

Thoracolaparoscopic esophagectomy (TLE) is comparable to conventional open esophagectomy in terms of oncological outcomes and with better short-term outcomes [3]. The major limiting factor of TLE is the technical complexity of the surgery especially in performing the thoracic phase of the surgery. The intricate relationship of esophagus with vital structures in the confined spaces of mediastinum could possibly make it an ideal structure to be tackled by the robotic surgical platform for all the benefits it can offer. Ever since the initial attempt at performing robotic esophagectomy in 2003 by Horgan and colleagues, a number of case series of robotic esophagectomy have been published 
from centers around the world with acceptable results [4, 5]. There are however very few studies comparing TLE and robot-assisted laparoscopic esophagectomy (RALE) for the management of esophageal cancer [6, 7]. No randomized controlled trial (RCT) data comparing RALE and TLE is available as of yet. Further data from the Indian subcontinent are sparse. This study aims to compare the short-term oncological outcomes following TLE and RALE through propensity-matched analysis.

\section{Methodology}

This is a retrospective propensity-matched analysis comparing TLE and RALE for carcinoma esophagus performed in a tertiary care teaching institute in India between January 2015 and September 2018 where over 30 cases of minimally invasive esophagectomy are performed annually. Data were gathered from the electronically stored hospital database. All the patients who underwent either TLE or RALE for carcinoma esophagus with curative intent were included in the study. Esophagectomy done for reasons other than carcinoma was excluded. Esophagectomy, primarily done by the open method, was also excluded.

The baseline parameters, including age, sex, tumor location, histopathological type, tumor stage, and details of neoadjuvant chemoradiotherapy (NACRT) were collected. Surgery related parameters namely type of surgery (TLE or RALE), the extent of surgery (Ivor-Lewis, Mc Keown), operative duration, blood loss, conversion to open procedure, resection margin status, lymph node yield, length of hospital stay and 90-day mortality rate were then compared between TLE and RALE. Further, to make the two groups more comparable and reduce the influence of confounding variables propensity matching was done for age, gender, BMI, ASA grade, tumor location, NACRT status, the extent of surgical resection (Ivor Lewis or McKeown's), histopathological type (squamous cell carcinoma or adenocarcinoma), clinical $\mathrm{T}$ and $\mathrm{N}$ stages. The primary outcome parameter was lymph node yield. The secondary outcome parameters were resection margin status, duration of surgery, blood loss, conversion to open procedure, length of hospital stay, length of ICU stay, complications, 90-day mortality and cost. Resection margin status was assessed by pathological examination for the presence of residual tumor. Absence of residual tumor cells was classified as R0, the presence of microscopic tumor at margins was classified as R1 and macroscopically visible tumor at margins was classified as R2 as per the College of American Pathologists criteria.

\section{Management protocol}

Patients diagnosed with carcinoma esophagus were evaluated with contrast-enhanced CT scan of the neck, chest, abdomen and pelvis. PET CT scan was done selectively if there was a high index of suspicion of metastasis like indeterminate lesions evident on CT. Tumors were staged according to AJCC 7 [8]. After discussion in the multidisciplinary tumor board, the plan for neoadjuvant therapy and surgery was made. Nutritional status of the patients were improved by enteral nutrition in the form of Ryle's tube feeding or feeding jejunostomy in patients receiving neoadjuvant therapy who had dysphagia severe enough to preclude adequate oral intake. Following neoadjuvant therapy, patients were reassessed with contrast-enhanced CT scan of the neck, chest, abdomen and pelvis to assess tumor response. All the patients were operated by only two surgeons. All RALE cases were done by the same surgeon while TLE cases were done by either of the two surgeons. The first surgeon (P.C) has performed over 750 TLE cases while the second surgeon (P.R) has performed over 45 TLE cases. The first surgeon started performing RALE after 2017. After 2017, the choice of approach was based primarily on patients' preferences. Post operative management was similar for all the patients irrespective of whether they had received TLE or RALE.

\section{Surgical technique}

\section{Thoracic part}

The thoracic part of the surgery was done under general anesthesia using single-lumen endotracheal tube with double lung ventilation. The patient was placed in semi-prone position. In TLE, a $10 \mathrm{~mm}$ camera port was placed in the 7th intercostal space (ICS) in the posterior axillary line. While introducing the initial trocar, tidal volume was reduced to about 150-200 ml. Pneumothorax was created using carbon dioxide to generate 8 to $10 \mathrm{mmHg}$ pressure facilitating the collapse of the right lung. The right hand working port was placed in the $5^{\text {th }}$ ICS and the left hand working port was placed in the 9th ICS, a little posterior to the posterior axillary line. An additional $5 \mathrm{~mm}$ port was placed in the 11th ICS in a few cases to facilitate dissection (Fig. 1). RALE was performed using da Vinci SI HD ${ }^{\mathrm{TM}}$ surgical system (Intuitive Surgical, Inc., CA, United States) In RALE, a $12 \mathrm{~mm}$ camera port was placed in the 6th ICS in the posterior axillary line. Robotic first arm was an $8 \mathrm{~mm}$ port in the $3 \mathrm{rd}$ ICS and the second arm was an $8 \mathrm{~mm}$ port in the 8th ICS. The first and second arms were posterior to the posterior axillary line. An optional $8 \mathrm{~mm}$ third arm was placed at the 10th ICS posterior to the posterior axillary line (Fig. 2). Diagnostic thoracoscopy was performed to assess the resectability. The triangular ligament was divided to retract the inferior lobe of the lung. The mediastinal pleura was then incised to expose the ventral aspect of the entire thoracic esophagus. The azygos vein was dissected and lifted from 


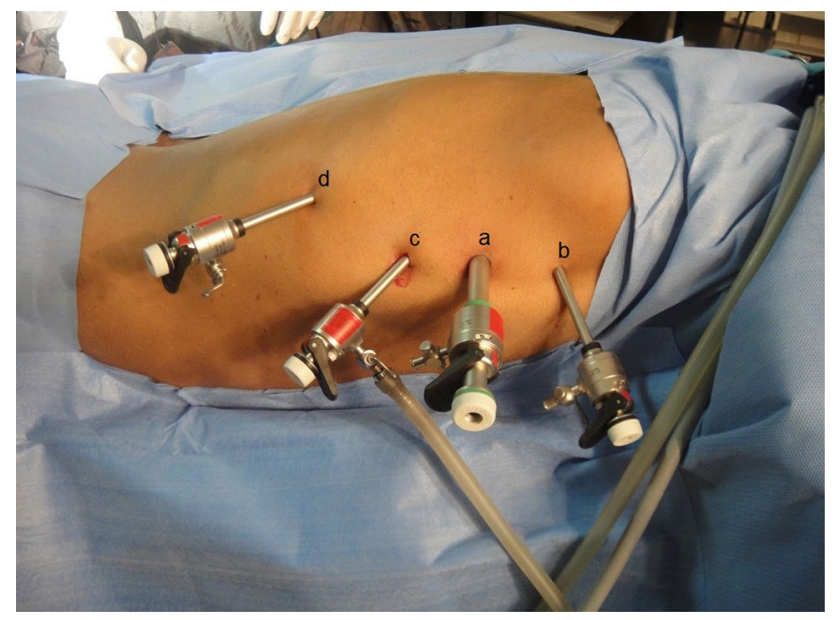

Fig. 1 Port position for the thoracic phase of TLE. a-Camera port in 7th ICS; b-right hand working port in 5th ICS; c-left hand working port in 9th ICS; d-optional $5 \mathrm{~mm}$ port in 11th ICS

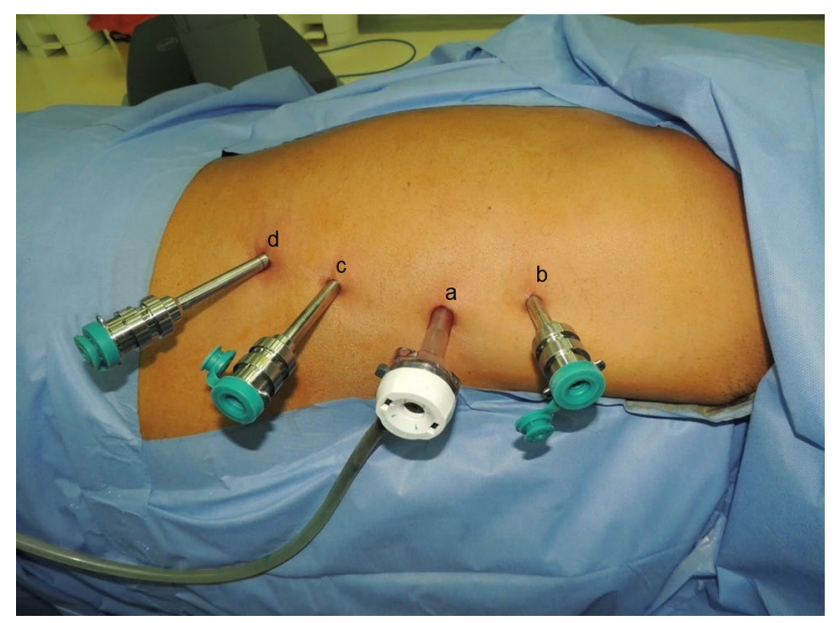

Fig. 2 Port position for the thoracic phase of RALE. a-Camera port in 6th ICS; b-robotic first arm in 3rd ICS; c-robotic second arm in 8th ICS; d-robotic third arm (optional) in 10th ICS

the underlying esophagus. The fully mobilized azygos vein was doubly ligated and divided. The thoracic esophagus was then mobilized circumferentially to include all periesophageal tissues from the esophageal hiatus to the thoracic inlet. An umbilical tape was used as a sling to facilitate dissection. Extended two field lymphadenectomy was done for both Ivor Lewis and McKeown's esophagectomy including para-esophageal, infracarinal, right para-tracheal and right recurrent laryngeal lymph nodes. In McKeowns's procedure, dissection ends at this point. In Ivor Lewis procedure, where abdominal dissection and conduit creation was done before thoracic dissection, specimen along with gastric conduit was pulled into the thoracic cavity. Esophagus was divided at the azygos arch level and specimen was taken out. Esophagogastric conduit anastomosis was then done using $25 \mathrm{~mm}$ circular stapler or by hand-sewn technique. The method of reconstruction was at the discretion of the operating surgeon. In some patients with financial constraints, hand-sewn anastomosis was chosen to bring down the cost. After ensuring hemostasis, a chest drain was placed in the right pleural cavity. Pneumothorax was deflated and port sites were closed.

\section{Abdominal part}

The abdominal phase of surgery was done in the supine position with leg split and 15 degree reverse Trendelenburg tilt. Pneumoperitoneum was created by Veress technique. In TLE, a $10 \mathrm{~mm}$ supraumbilical camera port was placed. A 5 or $10 \mathrm{~mm}$ right-hand working port was placed in the left midclavicular line. Left hand working port was a $12 \mathrm{~mm}$ port in the right mid-clavicular line. A $5 \mathrm{~mm}$ epigastric port was used for liver retraction and a $5 \mathrm{~mm}$ port was placed in the left anterior axillary line infra umbilically for gastric retraction (Fig. 3). In RALE, a $12 \mathrm{~mm}$ camera port was placed in the umbilical region. Robotic first arm was an $8 \mathrm{~mm}$ port placed in the left mid-clavicular line while the second arm was an $8 \mathrm{~mm}$ port placed in the right mid-clavicular line. Robotic third arm was placed in the right anterior axillary line. A $12 \mathrm{~mm}$ laparoscopic port was used in the right infraumbilical region for use by the assisting surgeon for clip application and firing endostapler (Fig. 4). Pars flaccida was divided and left gastric pedicle identified. Left gastric artery and vein were ligated separately at the root after clearing the lymph nodes. All lymphofatty tissue over the celiac axis, common hepatic artery and proximal splenic artery were removed. Greater curvature of the stomach was

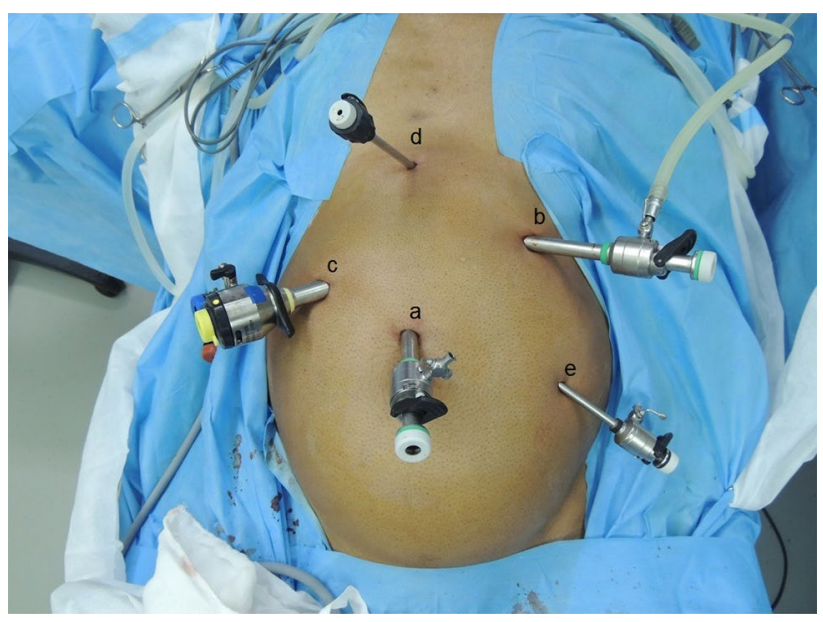

Fig. 3 Port position for the abdominal phase of TLE. a-Supra umbilical camera port; b-right hand working port in left mid clavicular line; c-left hand working port in right mid clavicular line; d-epigastric portfor liver retraction; e-port for gastric retraction in left anterior axillary line 


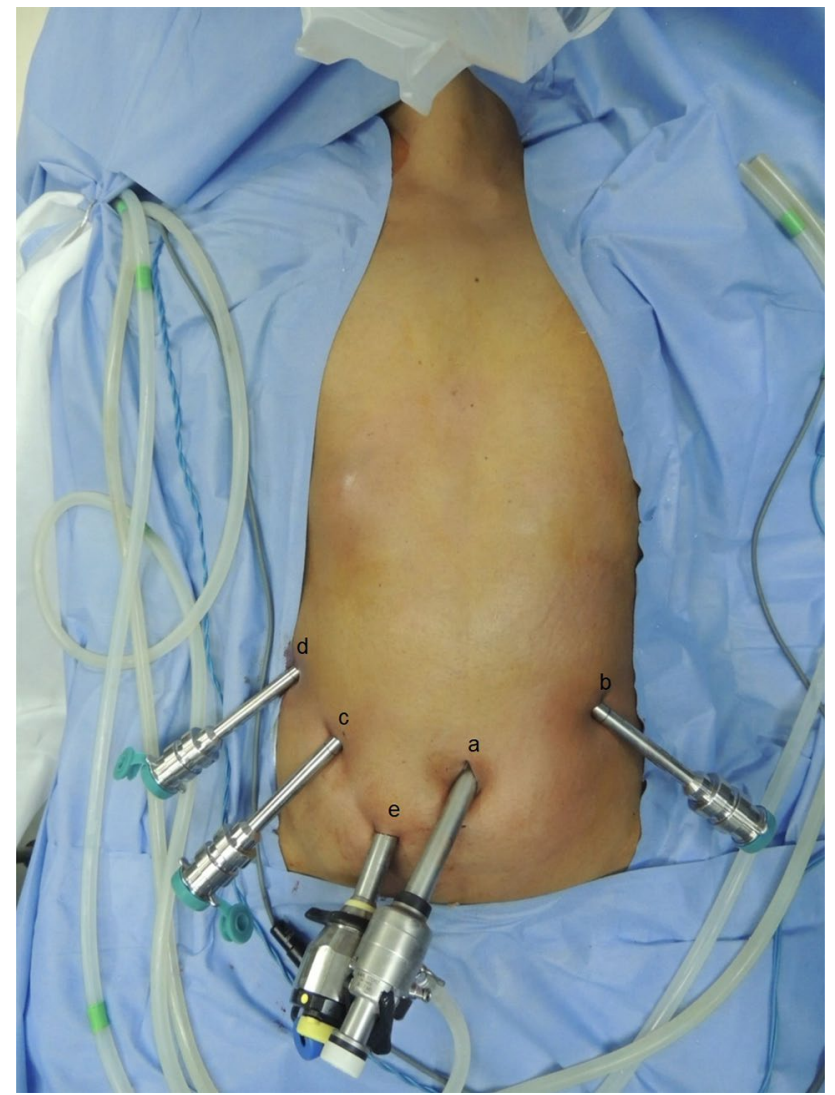

Fig. 4 Port position for the abdominal phase of RALE. a-Umbilical camera port; b-robotic first arm in left mid clavicular line; c-robotic second arm in right mid clavicular line; $d$-robotic third arm in rightanterior axillary line; e-laparoscopic $12 \mathrm{~mm}$ assistant port

mobilized from fundus to antrum by dividing gastrocolic omentum safeguarding the gastroepiploic arcade. Lower end of esophagus was completely mobilized. Stomach was divided $5 \mathrm{~cm}$ away from esophagogastric junction. Conduit was created by the serial firing of linear endostaplers forming a $5 \mathrm{~cm}$ wide gastric conduit.

\section{Neck part}

In McKeown's esophagectomy, the neck portion of surgery was done in the supine position with the neck extended and turned to the right. Left supraclavicular skin crease incision was made. Platysma was divided, strap muscles were separated and esophagus was identified. After mobilizing all around, esophagus was transected and specimen taken out in a plastic bag through neck incision by railroad technique. Gastric conduit was pulled up in the neck without any undue traction or twist. Esophagogastric conduit anastomosis was then done using $45 \mathrm{~mm}$ linear stapler or by hand-sewn technique. Neck incision was closed after keeping a small corrugated drain in the peri-anastomotic area.

\section{Statistical analysis}

Continuous variables were expressed as mean \pm standard deviation while categorical variables were expressed as proportions. Propensity score analysis was performed using $\mathrm{R}$ software version 4.0.3 and $\mathrm{R}$ studio version 1.3.1093. The analysis was carried out in Mac platform (x86_64-appledarwin17.0). Propensity score was generated using a multivariate logistic regression model with covariates namely age, gender, BMI, ASA grade, tumor location, NACRT status, the extent of surgical resection (Ivor Lewis or McKeown's), histopathological type (squamous cell carcinoma or adenocarcinoma), clinical $\mathrm{T}$ and $\mathrm{N}$ stages. Patients who underwent RALE and TLE were matched 1:1 using the nearest neighbor method with a caliper width of 0.02 using Matching package in R software.

The statistical analysis was performed using IBM SPSS Statistics Subscription (Build 1.0.0.1461; 64-bit edition). Independent $t$ test was used for continuous variables and chi-squared test was used for comparison of categorical variables. A two-sided $p$-value of less than 0.05 was considered statistically significant.

\section{Results}

There were 115 patients with esophageal cancer who underwent either TLE or RALE between January 2015 and September 2018. Of those patients, 90 underwent TLE, and 25 underwent RALE. After propensity matching there were 22 patients in each group. The baseline characteristics before and after matching are shown in Table 1 . The lymph node yield was higher in the RALE group, but it was not statistically significant. The operative duration was significantly longer in the RALE group both before and after matching. There was no conversion to open procedure in both the groups, and resection margins were free of tumor invasion in all the patients in both groups. The operative and short-term oncological outcome parameters before and after matching are shown in Table 2. Chyle leak was seen in one patient in both the groups, while anastomotic leak was seen in three patients in the TLE group before propensity matching. The cost of surgery was significantly higher in the RALE group both before and after matching. The postoperative complications and cost analyses are depicted in Table 3 .

\section{Discussion}

Minimally invasive esophagectomy has been continuously evolving over the last two decades. It is increasingly adopted by surgeons in the management of esophageal cancer with the hope of lowering the morbidity associated with 
Table 1 Baseline demographic and tumor characteristics

\begin{tabular}{|c|c|c|c|c|c|c|}
\hline Parameter & TLE $(n=90)$ & RALE $(n=25)$ & $p$ value & $\operatorname{TLE}_{\mathrm{m}}(n=22)$ & $\operatorname{RALE}_{\mathrm{m}}(n=22)$ & $p$ value \\
\hline $\begin{array}{l}\text { Age (years) } \\
\text { Mean } \pm \text { SD }\end{array}$ & $64.51 \pm 12.21$ & $61.88 \pm 9.83$ & 0.324 & $59.27 \pm 11.60$ & $60.91 \pm 9.31$ & 0.609 \\
\hline Males $n(\%)$ & $52(57.78 \%)$ & $17(68 \%)$ & 0.351 & $12(57.14 \%)$ & $14(63.64 \%)$ & 0.539 \\
\hline $\begin{array}{l}\text { BMI }\left(\mathrm{kg} / \mathrm{m}^{2}\right) \\
\text { Mean } \pm \mathrm{SD}\end{array}$ & $18.22 \pm 2.14$ & $20.32 \pm 4.24$ & $0.001 *$ & $18.91 \pm 2.07$ & $19.07 \pm 2.08$ & 0.797 \\
\hline \multicolumn{7}{|c|}{ ASA grade $n(\%)$} \\
\hline 1 & $2(2.22 \%)$ & 0 & \multirow[t]{3}{*}{0.586} & $2(9.09 \%)$ & 0 & \multirow[t]{3}{*}{0.211} \\
\hline 2 & $59(65.56 \%)$ & $16(64 \%)$ & & $15(68.18 \%)$ & $15(68.18 \%)$ & \\
\hline 3 & $29(32.22 \%)$ & $9(36 \%)$ & & $5(22.73 \%)$ & $7(31.82 \%)$ & \\
\hline \multicolumn{7}{|c|}{ Tumor location $n(\%)$} \\
\hline Upper 3rd & $1(1.11 \%)$ & 0 & \multirow[t]{4}{*}{0.209} & $1(4.55 \%)$ & 0 & \multirow[t]{4}{*}{0.560} \\
\hline Middle 3rd & $14(15.56 \%)$ & $7(28 \%)$ & & $5(22.73 \%)$ & $6(27.27 \%)$ & \\
\hline Lower 3rd & $16(17.78 \%)$ & $7(28 \%)$ & & $4(18.18 \%)$ & $6(27.27 \%)$ & \\
\hline GEJ & $59(65.56 \%)$ & $11(44 \%)$ & & $12(54.55 \%)$ & $10(45.45 \%)$ & \\
\hline \multicolumn{7}{|c|}{ Histology $n(\%)$} \\
\hline $\mathrm{SCC}$ & $58(64.44 \%)$ & $19(76 \%)$ & \multirow[t]{2}{*}{0.267} & $17(77.27 \%)$ & $17(77.27 \%)$ & \multirow[t]{2}{*}{1.000} \\
\hline $\mathrm{AC}$ & $32(35.56 \%)$ & $6(24 \%)$ & & $5(22.73 \%)$ & $5(22.73 \%)$ & \\
\hline \multicolumn{7}{|c|}{ Tumor grade $n(\%)$} \\
\hline 1 & 0 & $2(8 \%)$ & \multirow[t]{3}{*}{$0.017^{*}$} & 0 & $2(9.09 \%)$ & \multirow[t]{3}{*}{0.120} \\
\hline 2 & $86(95.56 \%)$ & $20(80 \%)$ & & $21(95.45 \%)$ & $17(77.27 \%)$ & \\
\hline 3 & $4(4.44 \%)$ & $3(12 \%)$ & & $1(4.55 \%)$ & $3(13.64 \%)$ & \\
\hline \multicolumn{7}{|l|}{ cT $n(\%)$} \\
\hline $1 / 2$ & $19(21.11 \%)$ & $6(24 \%)$ & \multirow[t]{2}{*}{0.759} & $7(31.82 \%)$ & $6(27.27 \%)$ & \multirow[t]{2}{*}{0.741} \\
\hline $3 / 4$ & $71(78.89 \%)$ & $19(76 \%)$ & & $15(68.18 \%)$ & $16(72.73 \%)$ & \\
\hline \multicolumn{7}{|l|}{$\mathrm{cN} n(\%)$} \\
\hline No & $36(40 \%)$ & $10(40 \%)$ & \multirow[t]{2}{*}{1.000} & $10(45.45 \%)$ & $9(40.91 \%)$ & \multirow[t]{2}{*}{0.761} \\
\hline $\mathrm{N}+$ & $54(60 \%)$ & $15(60 \%)$ & & $12(54.55 \%)$ & $13(59.09 \%)$ & \\
\hline \multicolumn{7}{|c|}{ Neoadjuvant therapy $n(\%)$} \\
\hline Yes & $51(56.67 \%)$ & $18(72 \%)$ & \multirow[t]{2}{*}{0.159} & $19(86.36 \%)$ & $17(77.27 \%)$ & \multirow[t]{2}{*}{0.432} \\
\hline No & $39(43.33 \%)$ & $7(28 \%)$ & & $3(13.64 \%)$ & $5(22.73 \%)$ & \\
\hline \multicolumn{7}{|l|}{ Surgery $n(\%)$} \\
\hline McKeown & $47(52.22 \%)$ & $15(60 \%)$ & \multirow[t]{2}{*}{0.489} & $12(54.55 \%)$ & $14(63.64 \%)$ & \multirow[t]{2}{*}{0.539} \\
\hline Ivor Lewis & $43(47.78 \%)$ & $10(40 \%)$ & & $10(45.45 \%)$ & $8(36.36 \%)$ & \\
\hline \multicolumn{7}{|c|}{ Anastomosis $n(\%)$} \\
\hline Hand sewn & $40(44.44 \%)$ & $3(12 \%$ & \multirow[t]{2}{*}{$0.002 *$} & $8(36.36 \%)$ & $3(13.64 \%$ & \multirow[t]{2}{*}{0.077} \\
\hline Stapled & $50(55.56 \%)$ & $22(88 \%)$ & & $14(63.64 \%)$ & $19(86.36 \%)$ & \\
\hline
\end{tabular}

$T L E$ thoracolaparoscopic esophagectomy, RALE robot assisted laparoscopic esophagectomy, BMI body mass index, ASA American Society of Anesthesiologists, GEJ gastroesophageal junction, $S C C$ squamous cell carcinoma, $A C$ adenocarcinoma, $c T$ clinical $\mathrm{T}$ stage, $c N$ clinical N stage, $S D$ standard deviation

*Statistically significant

conventional open esophagectomy [9]. TLE is, however, a technically complex surgery with a steep learning curve and is performed only by surgeons who are experienced in thoracoscopic, laparoscopic as well as esophageal surgeries [10]. Robotic surgery has several benefits over laparoscopic surgery like 3D vision, articulating instruments with a 7-degree range of movement and tremor filter. All these features appear to make RALE an attractive alternative to TLE. This study was performed to identify if the short-term oncological and perioperative outcomes following RALE were better than that following TLE.

The total duration of surgery was significantly longer in the robotic group than in the TLE group in our study. This could be because of our greater experience with TLE than with RALE. Similar findings were also observed by $\mathrm{He}$ et al. [11] (349 vs. $294 \mathrm{~min} ; p<0.001$ ) and Deng et al. [12] (354 vs. $284 \mathrm{~min} ; p<0.001$ ). Yang et al. [13], on the other hand, found that the overall surgery ( $244.5 \mathrm{vs} .276 .0 \mathrm{~min}$, 
Table 2 Operative and short-term oncological outcome parameters

\begin{tabular}{lllcccc}
\hline Parameter & TLE $(n=90)$ & RALE $(n=25)$ & $p$-value & TLEm $(n=22)$ & RALEm $(n=22)$ & $p$-value \\
\hline Total LN & $23 \pm 9.67$ & $24.72 \pm 8.97$ & 0.426 & $22.73 \pm 11.63$ & $23.95 \pm 8.23$ \\
Duration (min) & $444 \pm 61.39$ & $525.40 \pm 93.70$ & $<0.001^{*}$ & $444.77 \pm 64.91$ & $513.18 \pm 91.23$ & 0.688 \\
Blood loss (ml) & $139.39 \pm 31.30$ & $138.20 \pm 31.72$ & 0.867 & $133.18 \pm 34.80$ & $138.86 \pm 31.20$ & $0.006^{*}$ \\
Hospital stay (days) & $14.09 \pm 7.53$ & $11.88 \pm 6.06$ & 0.180 & $12.73 \pm 7.83$ & $12.18 \pm 6.35$ \\
ICU stay (days) & $5.10 \pm 4.61$ & $4.60 \pm 4.96$ & 0.638 & $4.77 \pm 4.81$ & $4.91 \pm 5.22$ \\
Mortality $n(\%)$ & $2(2.22 \%)$ & 0 & 0.320 & $1(4.55 \%)$ & 0 & 0.801 \\
\hline
\end{tabular}

$T L E$ thoracolaparoscopic esophagectomy, $R A L E$ robot asssited laparoscopic esophagectomy, $L N$ lymph node, ICU intensive care unit

*Statistically significant

Table 3 Post operative complications and cost analyses

\begin{tabular}{|c|c|c|c|c|c|c|}
\hline Parameter & TLE $(n=90)$ & $\operatorname{RALE}(n=25)$ & $p$-value & TLEm $(n=22)$ & RALEm $(n=22)$ & $p$-value \\
\hline Pneumonia $n(\%)$ & $8(8.89 \%)$ & $3(12 \%)$ & 0.648 & $4(18.18 \%)$ & $3(13.64 \%)$ & 0.680 \\
\hline RLN paresis $n(\%)$ & $7(7.78 \%)$ & $3(14.29 \%)$ & 0.533 & $1(4.55 \%)$ & $3(13.64 \%)$ & 0.306 \\
\hline Chyle leak $n(\%)$ & $1(1.11 \%)$ & $1(4.76 \%)$ & 0.375 & $1(4.55 \%)$ & $1(4.55 \%)$ & 1.000 \\
\hline Anastomotic leak $n(\%)$ & $3(3.33 \%)$ & 0 & 0.222 & $1(4.55 \%)$ & 0 & 0.235 \\
\hline Clavien Dindo (>3a) $n(\%)$ & $8(8.89 \%)$ & $3(12 \%)$ & 0.648 & $2(9.09 \%)$ & $3(13.64 \%)$ & 0.634 \\
\hline Cost $(\$)$ & $4407.50 \pm 570.36$ & $5258.53 \pm 431.67$ & $<0.001 *$ & $4243.01 \pm 474.64$ & $5271.75 \pm 456.46$ & $<0.001 *$ \\
\hline
\end{tabular}

$T L E$ thoracolaparoscopic esophagectomy, $R A L E$ robot assisted laparoscopic esophagectomy, $R L N$ recurrent laryngeal nerve, $\$$ United States Dollars

*Statistically significant

$p<0.001)$ as well as the thoracic part of the operation (85.0 vs. $102.9 \mathrm{~min}, p<0.001$ ) was faster in the robotic group.

The intraoperative blood loss was similar in both the groups in our study, as it was in multiple other studies [6, 14, 15]. In the study by Deng, however, blood loss was significantly lower in the robotic group (97 vs. $161 \mathrm{ml} ; p=0.015$ ) [12]. The lengths of hospital stay and ICU stay were not different between the two groups in our study. Comparable hospital and ICU stay was also reported by Chen et al. [16]. The median hospital stay was also similar in both the groups in the study by Zhang et al. [17] ( $p=0.517)$.

Achieving R0 resection is critical to have favorable oncological outcomes. Despite the several advantages of robotic surgery, which could facilitate easier dissection in confined spaces of the mediastinum, no difference in $\mathrm{R} 0$ resection was seen between RALE and TLE. Harbison et al. analyzed the American College of Surgeons National Surgical Quality Improvement Program database and found that R0 resection rates were comparable between RALE and TLE [18]. In a study by Weksler et al. [19] that analyzed the National Cancer Database, R0 resection was better with RALE than TLE (standardized difference-10.8\%; $p=0.027$ ). However, this difference disappeared after propensity matching $(p=0.688)$. Meredith et al., on the other hand, found a significant difference favoring RALE. R0 resection was achieved in $100 \%$ of the cases in RALE group while positive resection margins were observed in $6.5 \%$ of patients in thoracolaparoscopic Ivor Lewis group and 3.2\% in transhiatal esophagectomy group $(p=0.01)$ [7].

One of the significant advantages cited in the literature is a high lymph node yield for RALE. Park et al. [6] did a retrospective study and found that the lymph node yield was significantly higher in those who underwent RALE (37.3 \pm 17.1 vs. $28.7 \pm 11.8 ; p=0.003)$. Deng et al. [12], in a prospective study comparing RALE and TLE, found a higher total lymph node yield ( 21.9 vs. $17.8 ; p=0.042$ ) and a higher lymph node yield along the right recurrent laryngeal nerve (RLN) (2.1 vs. $1.2 ; p=0.033)$ in the robotic group. In a propensity-matched analysis done by Deng et al. [14], they noticed a higher total lymph node yield $(21.5 \pm 8.4 \mathrm{vs}$. $17.3 \pm 6.5 ; p=0.006$ ) as well as a higher lymph node yield along the left RLN $(1 \pm 1.8$ vs. $0.4 \pm 0.8 ; p=0.033)$ in the robotic group. Though the lymph node yield was comparable for the two groups, Chao et al. [15] found a significantly higher lymph node yield along the left RLN in the robotic group. In our study, though the lymph node yield was higher in the RALE group, it did not reach statistical significance. We did not, however, do a subgroup analysis of the lymph node yield specifically along the RLN, which was reported to be significantly higher in the robotic group in several studies. Our finding was in concordance with that reported by He et al. [11] (20 vs. 19; $p=0.420)$ and Weksler et al. [20] 
(19 vs. $22 ; p=0.950)$. Jin et al. [21] in their meta-analysis of eight case-controlled studies found no difference in lymph node yield between RALE and TLE.

Dissection to retrieve nodes along RLN puts the nerve at risk of injury. RLN injury leading to vocal cord palsy was similar in both the groups before and after matching in our study. Our findings were consistent with those reported by several other authors $[11,12,20]$. On the contrary, Yang et al. [13] published a higher incidence of RLN injury with robotic surgery $(29.2 \%$ vs. $15.1 \%, p<0.001)$. A meta-analysis data, however, showed that TLE was associated with higher rates of vocal cord palsy than RALE $(\mathrm{OR}=0.5696$, $p=0.0447$ ) [21]. A higher lymph node yield with lower rates of RLN injury with RALE, if confirmed by further prospective studies, can be viewed as a significant advantage. Chyle leak was noted in two patients, one in each group. Thoracoscopic ligation of the thoracic duct was done in the patient who underwent TLE following failed conservative trial while the patient in the RALE group responded to conservative management. The overall complication rates, as well as complications requiring intervention (Clavien-Dindo grade IIIa and above), were similar in both arms of our study. Park et al. [6] have also reported similar outcomes in terms of Clavien-Dindo grading and other complications. Analysis of an extensive surgical database also failed to show any difference between RALE and TLE in terms of complications [18].

Anastomotic leak is a dreaded complication of esophagectomy, and though mortality rates have come down significantly, it is still associated with high morbidity [22]. Factors affecting the integrity of the anastomosis include patientrelated factors like BMI, treatment-related factors like neoadjuvant therapy, and surgical factors like location of the anastomosis (cervical versus intrathoracic) [23]. We compared the leak rates between RALE and TLE both before and after matching for these factors and found no difference between the two groups. In the propensity-matched analysis by Tagkalos et al. [24], similar leak rates were seen both before $(18 \%$ vs. $12 \% ; p=0.575)$ and after matching $(12.5 \%$ vs. $12.5 \% ; p=0.966)$. They, however, had included only patients who underwent modified Ivor-Lewis esophagectomy while we included both Ivor-Lewis and McKeown esophagectomy patients.

There were two deaths within 90 days in the TLE group, while there was no mortality in the RALE group. Anastomotic leak was the underlying cause for death in both the patients and the pre-operative ASA category was 3 in both of them. One of the patients was treated with esophageal stent initially, which migrated and later required thoracoscopic lavage. The other patient was treated by thoracoscopic lavage and mediastinal drainage. Further course was complicated by ventilator-associated pneumonia and multi-organ dysfunction in both the cases. The 90-day mortality was, however, comparable between RALE and TLE both before and after matching. In a study comparing robot-assisted and thoracoscopic assisted Ivor Lewis esophagectomy, Zhang et al. [17] reported no deaths within 30 days and comparable 90-day mortality between the two groups (1.3\% vs. $1.9 \%$; $p=1)$. Chao et al. [15], in their study comparing robotassisted and video-assisted McKeown's esophagectomy, also reported no 30-day mortality and comparable 90-day mortality $(2.7 \%$ vs. $3.8 \% ; p=1)$. A meta-analysis involving 1862 patients also confirmed these findings of comparable 30-day $(p=0.76)$ and 90 -day mortality rates $(p=0.33)$ [21]. The costs involved in the management of patients who underwent RALE were significantly higher than the TLE group in our study. This is despite the comparable complication rates, ICU and hospital stay in both the groups, reflecting the higher costs involved in the procurement and maintenance of the robotic system.

The strength of this study is attributed to propensity matching that was done to make the robotic and thoracolaparoscopic groups more comparable and possibly eliminate the known confounding factors. Further, this is the first study from the Indian subcontinent comparing RALE and TLE in the management of carcinoma esophagus. This study is, however, not devoid of limitations. First, being a retrospective study, the data collected are subject to inherent limitations of any retrospective analysis. As the robotic platform was not available initially, there is a possibility of selection bias. Second, the small sample size in the matched cohort could mean that some of the results may not be truly reflective of the actual outcomes. Third, specific details about lymph node yield along RLN were not available for analysis. Fourth, the greater experience of the operating surgeons in TLE and limited experience in RALE could have affected the results in favor of TLE.

The small sample size in our study could have been the reason why the difference in lymph node yield did not reach statistical significance. With recent evidence pointing to better survival outcomes in patients with higher lymph node yield following esophagectomy, identifying the technique that offers better lymph node yield is the need of the hour [25]. Future RCTs comparing RALE and TLE should therefore focus on lymph node yield, especially along the RLN. This can help identify if RALE can contribute to better lymph node yield without increasing the risk of RLN injury and possibly contribute to better survival.

\section{Conclusion}

Both RALE and TLE are equally effective in the management of carcinoma esophagus. The short-term oncological outcomes and complications following RALE and TLE for 
the management of carcinoma esophagus are comparable. RALE is associated with longer operating duration and increased costs than TLE.

Acknowledgements The authors would like to acknowledge the assistance provided by Mr Karthik M, 2nd year MBA, Department of Management Studies, NIT-Tiruchirappali for statistical analysis.

Funding No funding was received for conducting this study.

\section{Compliance with ethical standards}

Conflict of interest Author 1, Author 2, Author 3, Author 4 and Author 5 declare that they have no conflicts of interest to declare that are relevant to the content of this article.

Ethics approval Ethical approval was waived by the Institutional Ethics Committee in view of the retrospective nature of the study and all the procedures being performed were part of the routine care.

\section{References}

1. Samarasam I (2017) Esophageal cancer in India: Current status and future perspectives. Int J Adv Med Heal Res 4:5-10. http:// www.ijamhrjournal.org/article.asp?issn=2349-4220

2. Mariette C, Markar S, Dabakuyo-Yonli TS, Meunier B, Pezet D, Collet D et al (2020) Health-related quality of life following hybrid minimally invasive versus open esophagectomy for patients with esophageal cancer, analysis of a multicenter, open-label, randomized phase iii controlled trial: the MIRO trial. Ann Surg 271:1023-1029

3. Verhage RJJ, Hazebroek EJ, Boone J, Van Hillegersberg R (2009) Minimally invasive surgery compared to open procedures in esophagectomy for cancer: a systematic review of the literature. Minerva Chir 64:135-146

4. Horgan S, Berger RA, Elli EF, Espat NJ (2003) Robotic-assisted minimally invasive transhiatal esophagectomy. Am Surg 69:624-626

5. Ruurda JP, van der Sluis PC, van der Horst S, van Hilllegersberg R (2015) Robot-assisted minimally invasive esophagectomy for esophageal cancer. A systematic review. J Surg Oncol 112:257-265

6. Park S, Hwang Y, Lee HJ, Park IK, Kim YT, Kang CH (2016) Comparison of robot-assisted esophagectomy and thoracoscopic esophagectomy in esophageal squamous cell carcinoma. J Thorac Dis 8:2853-2861

7. Meredith K, Blinn P, Maramara T, Takahashi C, Huston J, Shridhar R (2020) Comparative outcomes of minimally invasive and robotic-assisted esophagectomy. Surg Endosc 34:814-820

8. Rice TW, Blackstone EH, Rusch VW (2010) 7th edition of the AJCC cancer staging manual: esophagus and esophagogastric junction. Ann Surg Oncol 17:1721-1724

9. Schumer E, Perry K, Melvin WS (2012) Minimally invasive esophagectomy for esophageal cancer: evolution and review. Surg Laparosc Endosc Percutan Tech 22:383-386

10. Wang Q, Wu Z, Chen G, Zhang S, Shen G, Wu M (2018) Twostage indicators to assess learning curves for minimally invasive ivor lewis esophagectomy. Thorac Cardiovasc Surg 66:362-369
11. He H, Wu Q, Wang Z, Zhang Y, Chen N, Fu J et al (2018) Shortterm outcomes of robot-assisted minimally invasive esophagectomy for esophageal cancer: a propensity score matched analysis. J Cardiothorac Surg 13:52

12. Deng H-Y, Huang W-X, Li G, Li S-X, Luo J, Alai G et al (2018) Comparison of short-term outcomes between robot-assisted minimally invasive esophagectomy and video-assisted minimally invasive esophagectomy in treating middle thoracic esophageal cancer. Dis Esophagu 31:8. https://doi.org/10.1093/dote/doy012

13. Yang Y, Zhang X, Li B, Hua R, Yang Y, He Y et al (2019) Shortand mid-term outcomes of robotic versus thoraco-laparoscopic McKeown esophagectomy for squamous cell esophageal cancer: a propensity score-matched study. Dis Esophagus 33(6):080. https ://doi.org/10.1093/dote/doz080

14. Deng H-Y, Luo J, Li S-X, Li G, Alai G, Wang Y et al (2019) Does robot-assisted minimally invasive esophagectomy really have the advantage of lymphadenectomy over video-assisted minimally invasive esophagectomy in treating esophageal squamous cell carcinoma? A propensity score-matched analysis based on shortterm. Dis Esophagus 32(7):doy110. https://doi.org/10.1093/dote/ doy 110

15. Chao YK, Hsieh MJ, Liu YH, Liu HP (2018) Lymph node evaluation in robot-assisted versus video-assisted thoracoscopic esophagectomy for esophageal squamous cell carcinoma: a propensity-matched analysis. World J Surg 42:590-598

16. Chen J, Liu Q, Zhang X, Yang H, Tan Z, Lin Y et al (2019) Comparisons of short-term outcomes between robot-assisted and thoraco-laparoscopic esophagectomy with extended two-field lymph node dissection for resectable thoracic esophageal squamous cell carcinoma. J Thorac Dis 11:3874-3880

17. Zhang Y, Han Y, Gan Q, Xiang J, Jin R, Chen K et al (2019) Early outcomes of robot-assisted versus thoracoscopic-assisted ivor lewis esophagectomy for esophageal cancer: a propensity score-matched study. Ann Surg Oncol 26:1284-1291

18. Harbison GJ, Vossler JD, Yim NH, Murayama KM (2019) Outcomes of robotic versus non-robotic minimally-invasive esophagectomy for esophageal cancer: an American College of Surgeons NSQIP database analysis. Am J Surg 218:1223-1228

19. Weksler B, Sullivan JL (2017) Survival after esophagectomy: a propensity-matched study of different surgical approaches. Ann Thorac Surg 104:1138-1146

20. Weksler B, Sharma P, Moudgill N, Chojnacki KA, Rosato EL (2012) Robot-assisted minimally invasive esophagectomy is equivalent to thoracoscopic minimally invasive esophagectomy. Dis Esophagus 25(5):403-409

21. Jin D, Yao L, Yu J, Liu R, Guo T, Yang K et al (2019) Roboticassisted minimally invasive esophagectomy versus the conventional minimally invasive one: a meta-analysis and systematic review. Int J Med Robot 15:e1988

22. Martin LW, Swisher SG, Hofstetter W, Correa AM, Mehran RJ, Rice DC et al (2005) Intrathoracic leaks following esophagectomy are no longer associated with increased mortality. Ann Surg 242:392-402

23. Kassis ES, Kosinski AS, Ross PJ, Koppes KE, Donahue JM, Daniel VC (2013) Predictors of anastomotic leak after esophagectomy: an analysis of the society of thoracic surgeons general thoracic database. Ann Thorac Surg 96:1919-1926

24. Tagkalos E, Goense L, Hoppe-Lotichius M, Ruurda JP, Babic B, Hadzijusufovic E et al (2020) Robot-assisted minimally invasive esophagectomy (RAMIE) compared to conventional minimally invasive esophagectomy (MIE) for esophageal cancer: a propensity-matched analysis. Dis Esophagus 33(4):doz060. https://doi. org/10.1093/dote/doz060 
25. Visser E, van Rossum PSN, Ruurda JP, van Hillegersberg R (2017) Impact of lymph node yield on overall survival in patients treated with neoadjuvant chemoradiotherapy followed by esophagectomy for cancer: a population-based cohort study in the Netherlands. Ann Surg 266:863-869
Publisher's Note Springer Nature remains neutral with regard to jurisdictional claims in published maps and institutional affiliations. 